\section{Carine Michiels Thierry Arnould José Remacle}

\title{
Rôle clé de l'hypoxie et des cellules endothéliales dans le développement des veines variqueuses
}

Les veines variqueuses représentent une anomalie fréquente dont l'étiologie n'est pas encore bien définie. Les études épidémiologiques ont montré qu'outre des facteurs génétiques et hormonaux, la station debout prolongée contribue à leur développement, peut-être par l'intermédiaire de l'hypoxie ainsi induite. Les molécules inflammatoires produites par les cellules endothéliales soumises à l'hypoxie stimulent en effet la prolifération des cellules musculaires lisses de la média et provoquent l'adhérence des polynucléaires neutrophiles. Ceux-ci, activés à leur tour, relarguent protéases et radicaux libres. Des phénomènes voisins sont retrouvés dans la paroi de la veine variqueuse. Certains composés qui inhibent la cascade d'activation des cellules endothéliales lors de l'hypoxie ont montré leur efficacité dans les troubles veineux. L'ensemble de ces données aboutit à formuler une hypothèse cohérente sur l'origine de l'insuffisance veineuse chronique provoquée par une ischémie locale.

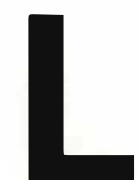

es veines variqueuses constituent un problème de santé majeur : environ une personne sur trois en souffre dans les pays occi-

C. Michiels : chargée de recherche du FNRS (Fonds national de la recherche scientifique, Bruxelles). T. Arnould : boursier du FNRS. J. Remacle : professeur de biochimie. Laboratoire de biochimie cellulaire, facultés universitaires Notre-Dame de la Paix, 61, rue de Bruxelles, 5000 Namur, Belgique.

\section{TIRÉS A PART}

\section{Michiels.}

et le régime alimentaire pauvre en fibres semblent être corrélés à la prévalence des varices au sein de la population mondiale $[1,3]$. La station debout prolongée est aussi incriminée : elle est bien connue comme facteur de risque pour le syndrome des jambes lourdes [3], mais aussi, selon certains auteurs, pour le développement des varices [1]. De plus, des perturbations mécaniques, comme des insuffisances des valvules dont le rôle est d'empêcher le reflux veineux, et une forte pression sanguine 


\section{RÉFÉRENCES}

1. Beaglehole R. Epidemiology of varicose veins. World J Surg 1986 ; 10 : 898-902.

2. Browse NL, Burnand KG. The cause of venous ulcerations. Lancet 1982 ; ii : 243-5.

3. Carpentier P, Priollet P. Épidémiologie de l'insuffisance veineuse chronique. Presse Med 1994 ; 23 : 197-201.

4. Niebes P. Structure biochimique de la paroi veineuse chez les porteurs de varices. Phlébologie 1983 ; 36 : 55-6.

5. Corvol P. L'endothélium, plaque tournante de la vasomotricité et de la trophicité de la paroi artérielle. médecine/sciences $1993 ; 9$ : 1031-3.

6. Borsum T. Biochemical properties of vascular endothelial cells. Virchows Arch B Cell Pathol 1991 ; 60 : 279-86.

7. Jaffe EA. Physiological functions of normal endothelial cells. Ann NY Acad Sci $1985 ; 454$ : 279-91.

8. Pearson JD. Endothelial cell biology. Radiology 1991 ; 179 : 9-14.

9. Van Obberghen-Schilling E, Pouysségur J. Le récepteur de la thrombine et ses implications dans la prolifération des cellules vasculaires. médecine/sciences 1993 ; $9: 1043-9$.

10. Springer TA. Adhesion receptors of the immune system. Nature $1990 ; 346: 25-434$.

11. Scozaec J, Lesèche G. Immunologie des cellules endothéliales et rejet de greffe. médecine/sciences $1993 ; 9$ : 1094-101.

12. Piper HM. Energy deficiency, calcium overload or oxidative stress : possible causes of irreversible ischemic myocardial injury. Klin Wochenschr 1989 ; 67 : 465-76.

13. Michiels C, Arnould T, Remacle J. Hypoxia-induced activation of endothelial cells as a possible cause of venous diseases: a hypothesis. Angiology 1993 : 639-46.

14. Michiels C, Arnould T, Houbion A, Remacle J. Human umbilical vein endothelial cells submitted to hypoxiareoxygenation in vitro: implication of free radicals, xanthine oxidase and energy deficiency. J Cell Physiol $1992 ; 153$ : 53-61. dans les veines, sont associées à leur apparition. Parallèlement, les études épidémiologiques ont montré qu'il faut aussi tenir compte des facteurs héréditaires. On relève, en effet, des antécédents familiaux chez $80 \%$ des sujets porteurs de varices. Certains travaux confortent l'hypothèse que le trait génétique concerne la transmission d'un tissu veineux de qualité médiocre [4].

Cependant, dans la plupart des cas où des veines variqueuses se développent, une stase sanguine est observée. Deux cas communs sont le reflux du sang des veines internes vers les veines externes et la réapparition des varices après une intervention chirurgicale quand une partie de la veine est toujours située en dehors du flux sanguin normal. La station debout prolongée est un facteur épidémiologique important qui est corrélé à l'apparition de la maladie et qui produit aussi une stase veineuse. On pourrait donc postuler que la stase du sang dans les veines des membres inférieurs est une des principales perturbations physiologiques conduisant au développement des veines variqueuses, mais aucun mécanisme biochimique n'a été proposé jusqu'à présent pour lier cette situation aux modifications tissulaires observées dans ces maladies. La clé de ce lien est, comme nous allons le voir, la réaction de l'endothélium à l'hypoxie.

L'endothélium constitue la première barrière entre le sang et le tissu veineux. Il est loin d'être inerte et remplit de nombreuses fonctions [5]. En tant que contenant hématocompatible, il contrôle la coagulation ainsi que l'activité des plaquettes par la libération de molécules anti-thrombiques comme la prostacycline $\left(\mathrm{PGI}_{2}\right)$, il synthétise des molécules vasoactives comme l'endothelium-derived relaxing factor (EDRF) et l'endothéline ainsi que de nombreux composés réglant l'activité des leucocytes comme le platelet-activating factor (PAF), l'interleukine 8 (IL8), la prostaglandine E2 $\left(\mathrm{PGE}_{\mathrm{o}}\right)$, les colonystimulating factors (CSF) et les facteurs de croissance qui influencent les cellules musculaires lisses (CML) comme le platelet-derived growth factor (PDGF) et l'endothelial cell-derived growth factor (ECDGF) [6-9]. L'endo- thélium agit aussi en barrière sélectivement perméable ; il sécrète de nombreux métabolites, notamment le collagène de type IV et la laminine qui sont des constituants de la matrice extracellulaire, et métabolise les lipoprotéines et l'angiotensine. Les cellules endothéliales interagissent également étroitement avec les leucocytes: ces interactions sont spécifiques de chaque type de leucocytes et sont finement réglées par l'induction de l'expression des molécules d'adhérence à la surface des cellules endothéliales et par la libération de facteurs chémotactiques $[10,11]$.

Lors d'une stase veineuse, l'apport en oxygène au tissu veineux est limité. Du fait de leur localisation à l'interface sang-tissu, les cellules endothéliales sont les premières à souffrir de cette situation hypoxique. Une réduction de la pression partielle en oxygène $\left(\mathrm{PO}_{2}\right)$ conduit à une diminution de l'activité de la chaîne respiratoire mitochondriale : les phosphorylations oxydatives ralentissent, ce qui diminue la régénération d'adénosine triphosphate (ATP) et, comme mécanisme de compensation, la glycolyse est stimulée. Si la cellule ne peut pas satisfaire tous ses besoins en ATP, les modifications métaboliques qui en résultent peuvent conduire à sa mort [12] . Cependant, avant que ne surviennent ces altérations irréversibles, l'hypoxie peut influencer les fonctions des cellules endothéliales, entraînant une cascade d'événements qui impliquent leucocytes et cellules musculaires lisses. Ce sont ces événements qui vont conduire aux modifications typiquement observées dans les parois veineuses pathologiques. Dans cet article, nous allons montrer comment les diverses étapes de ce processus séquentiel qui implique différents types cellulaires et dont l'origine est une réduction de l'apport en oxygène permettent de comprendre le développement de la pathologie veineuse [13].

\section{Arguments expérimentaux}

Le modèle expérimental sur lequel l'effet de l'hypoxie a été étudié est constitué d'une primo-culture de 


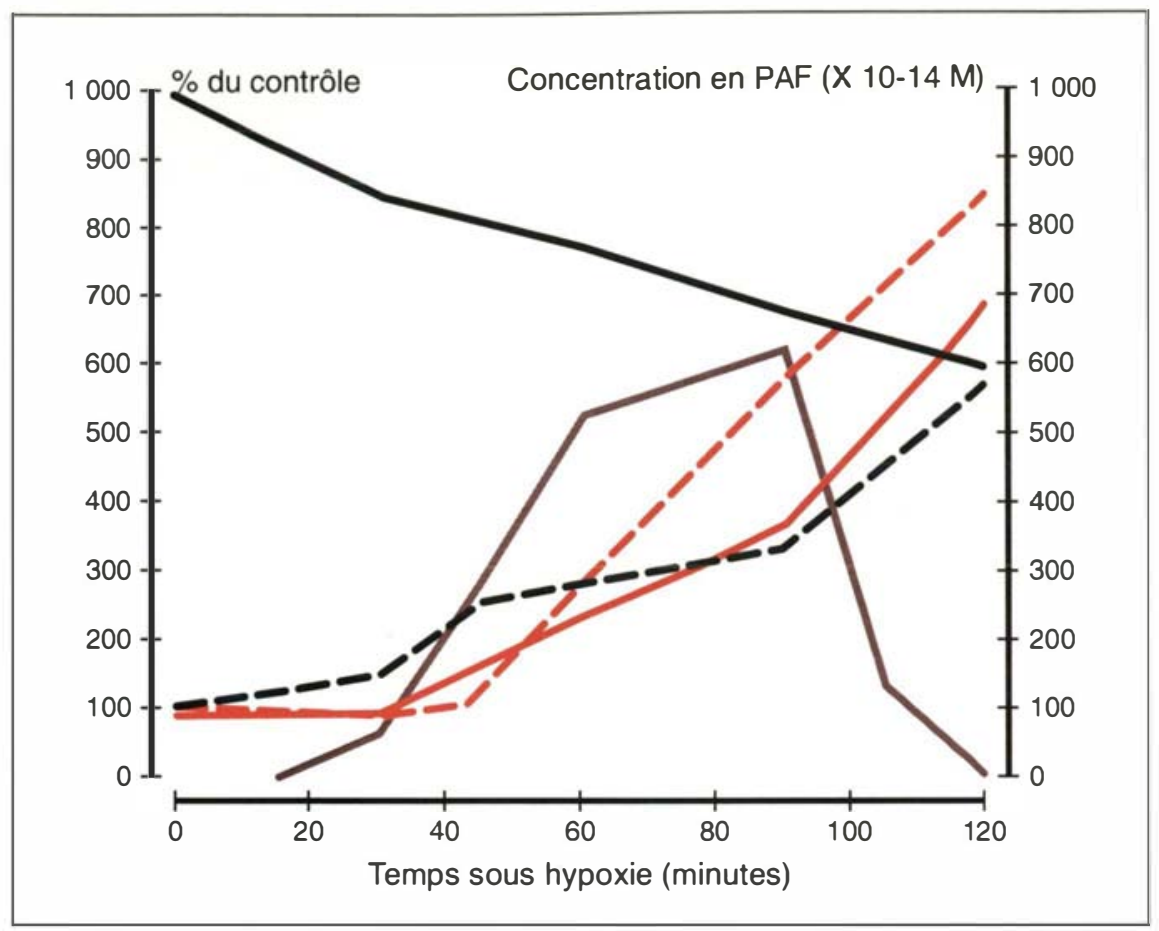

Figure 1. Cinétique des variations des paramètres biologiques des cellules endothéliales isolées à partir de veine ombilicale humaine (HUVEC) exposées à une hypoxie. ATP: courbe noire; concentration cytosolique en calcium : courbe rouge; synthèse de prostaglandines : courbe noire discontinue; synthèse de PAF : courbe bistre; augmentation de l'adhérence des $P M N$ aux HUVEC : courbe rouge discontinue. Les résultats sont exprimés en pourcentage (ou pour mille (ATP)) par rapport au contrôle maintenu en normoxie, sauf pour la synthèse de PAF qui est exprimée en valeurs absolues.

cellules endothéliales isolées à partir de la veine ombilicale humaine (HUVEC, human umbilical vein endothelial cells) exposées in vitro à une hypoxie sévère. Dans les conditions expérimentales de ce modèle, la pression partielle en oxygène atteint $10 \mathrm{~mm} \mathrm{Hg}$ dans le milieu après 15 minutes [14]. Dans ces conditions, on n'observe pas de mort cellulaire pendant les deux premières heures d'hypoxie. La mort cellulaire est cependant provoquée par des temps plus longs d'incubation sous hypoxie: il n'y a plus que $50 \%$ des cellules qui survivent après 6 heures d'hypoxie.

Durant l'hypoxie, la première modification métabolique observée est la diminution de la concentration en ATP résultant de la diminution des phosphorylations mitochondriales due au déficit en oxygène : une diminution de $43 \%$ de la concentration en ATP est enregistrée dans les lipase $A_{2},\left(P L A_{2}\right)$ remplit une fonction importante dans les cellules endothéliales : elle est, en effet, à l'origine de la synthèse de médiateurs pro-inflammatoires. Nous avons montré que la PLA, était activée lorsque les HUVEC étaient incubées sous hypoxie [16] ; cette activité conduit au relarguage d'acide arachidonique et de lyso-PAF à partir des phospholipides membranaires L'acide arachidonique est ensuite transformé en prostaglandines (PG) dans les cellules endothéliales, principalement par la cyclooxygénase. Une très forte induction de la synthèse de $P G$, jusqu'à six fois, est mesurée lorsque les HUVEC sont incubées 2 heures sous hypoxie : dans ces conditions, les HUVEC synthétisent $139,2,34,8,12,0$ et $9,5 \mathrm{ng}$ de $\mathrm{PG} / \mathrm{mg}$ de protéines respectivement pour la 6-céto-PGF (métabolite stable de la $\mathrm{PGI}_{2}$ ), la $\mathrm{PGF}_{2 \alpha}$, la

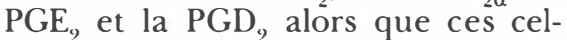
lules ne synthétisent respectivement que $26,9,7,7,1,4$ et $1,0 \mathrm{ng}$ de $\mathrm{PG} / \mathrm{mg}$ de protéines en conditions normales [16]. Le lyso-PAF est, quant à lui, transformé en PAF par l'acétyltransférase. En conditions normales, les cellules endothéliales ne synthétisent pas de PAF. Cependant, pendant l'incubation sous hypoxie et par suite de l'activation de la PLA, une importante production de PAF par les HUVEC est mesurée, conduisant à une concentration de $0,24.10^{-12}$ moles $/ 10^{6}$ cellules [17]. Le PAF n'est pas libéré dans le milieu et reste associé aux cellules. Une parfaite corrélation est observée entre les cinétiques de l'élévation de la concentration cytosolique en calcium, de l'activation de la phospholipase $A_{\text {, }}$, de la synthèse des prostaglandines et de la synthèse de PAF, indiquant qu'il s'agit bien d'un processus unique. Ce parallélisme entre les cinétiques de ces différents processus est illustré par la figure 1.

Ces résultats montrent que l'hypoxie est capable d'activer fortement les cellules endothéliales, conduisant à la libération de différents facteurs inflammatoires (figure 2). Ces divers médiateurs peuvent ensuite interagir avec d'autres types cellulaires. Dans la paroi veineuse, les cellules endothéliales sont principalement en contact avec deux types cellulaires 


\section{RÉFÉRENCES}

15. Arnould T, Michiels C, Alexandre I Remacle J. Effect of hypoxia upon intracellular calcium concentration of human endothelial cells. J Cell Physiol 1992 ; $152: 215-21$.

16. Michiels C, Arnould T, Knott I, Dieu M, Remacle J. Stimulation of prostaglandin synthesis by human endothelial cells exposed to hypoxia. Am J Physiol $1993 ; 264$ : C866-74.

17. Arnould T, Michiels C, Remacle J. Increased PMN adherence on endothelia cells after hypoxia : involvement of PAF CD18/CD11b and ICAM-1. Am J Physiol 1993 ; 264 : C1 102-10.

18. Pober JS, Cotran RS. The role of endothelial cells in inflammation. Transplantation $1990 ; 50: 537-44$

19. Zimmerman GA, McIntyre TM, Mehra M, Prescott SM. Endothelial cellsassociated platelet-activating factor: a nove mechanism for signaling intercellular adhesion. J Cell Biol $1990 ; 110$ : 529-40.

20. Arnould T, Michiels C, Remacle J Activation of adherent PMN to hypoxic HUVEC leads to cytotoxicity. Blood 1994 (sous presse).

21. Michiels C, De Leener F, Arnould T, Dieu M, Remacle J. Hypoxia stimulates human endothelial cells to release smooth muscle cell mitogens: role of prostaglandins. Exp Cell Res 1994 (sous presse).

22. Montefort S, Holgate ST. Adhesion molecules and their role in inflammation. Respir Med 1991 ; 85 : 91-9.

23. Niebes P. In : New trends in venous diseases, vol I. Bern : Hens Hubert Publishers, 1977 : 2231.

24. Borel JP, Monboisse JP, Bellon G Inflammation, collagène et radicaux libres oxygénés. médecine/sciences $1988 ; 4: 304-10$.

25. Charles A, Gresham GA. Histopathological changes in venous grafts and in varicose and non-varicose veins. J Clin Pathol 1993 ; 46 : 603-6.

26. Chamley-Campbell J, Campbell GR, Ross R. The smooth muscle cells in culture Physiol Rev $1979 ; 59: 1-61$.

27. Yamamoto M, Fujita K, Shinkai T Yamamoto $K$, Noumura T. Identification of the phenotypic modulation of rabbit arterial smooth muscle cells in primary culture by flow cytometry. Exp Cell Res 1992 $198: 43-51$.

28. Okada Y, Katsuda S, Matsui Y Watanabe $\mathrm{H}$, Nakanishi I. Collagen synthesis by cultured arterial smooth muscle cells during spontaneous phenotypic modu-

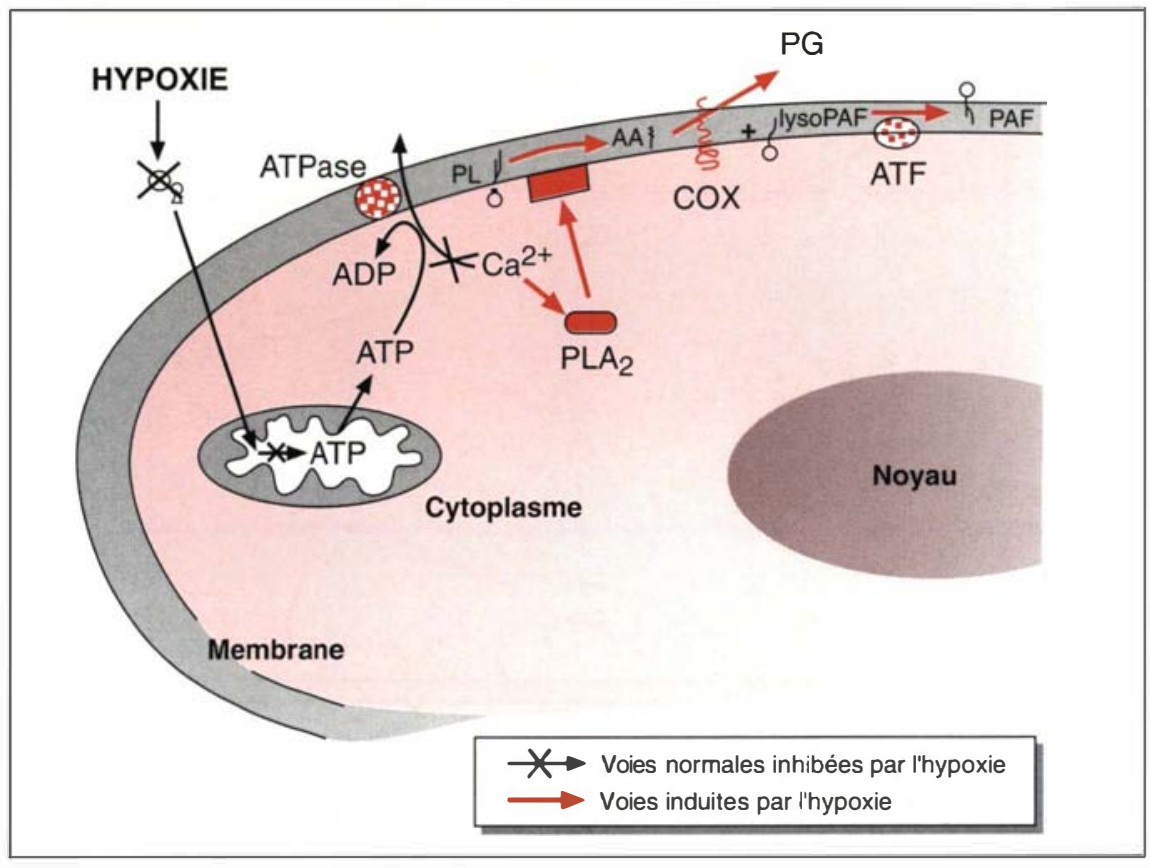

Figure 2. Schéma des principales réactions à l'hypoxie des cellules endothéliales. Les réactions sont indiquées suivant leur suite logique par des flèches montrant les réactions inhibées ou activées après hypoxie. Le Ca2+ cytosolique est augmenté par inhibition de la pompe calcique (déplétion de I'ATP) et le relarguage à partir des organites. II transmet le signal hypoxique (second messager) par activation de la phospholipase $A_{2}$ (PLA $)$. $L$ 'acide arachidonique (AA) produit à partir des phospholipides (PL) est transformé en de nombreuses molécules inflammatoires. COX: cyclooxygénase, PG : prostaglandine, Iyso-PAF, lyso-platelet-activating factor, ATF : acétyltransférase, PAF : platelet-activating factor.

les cellules musculaires lisses (CML) sous-jacentes qui constituent les principales cellules de la média et sont responsables du tonus vasculaire et les leucocytes circulant dans le sang. Parmi ceux-ci, les polymorphonucléaires neutrophiles (PMN) sont les plus abondants et ils sont sensibles aux médiateurs inflammatoires [18]. L'étape suivante des travaux était donc d'étudier comment l'hypoxie pouvait influencer les interactions entre les cellules endothéliales, d'une part, et les neutrophiles ou les cellules musculaires lisses, d'autre part.

L'adhérence des PMN aux cellules endothéliales est très faible dans des conditions normales, mais elle devient plus importante lorsque les PMN ou les cellules endothéliales sont activés. On observe que l'adhérence des PMN humains non stimu- lés aux HUVEC est augmentée si ces cellules ont été préalablement incubées sous hypoxie, et ce, d'autant plus que l'incubation sous hypoxie est longue (figure 1) [18]. Cette adhérence est directement induite par le PAF présent à la surface des HUVEC ayant subi une incubation de 90 minutes sous hypoxie : elle est, en effet, complètement inhibée si la synthèse de PAF par les HUVEC est inhibée ou si la mesure de l'adhérence se fait en présence d'un antagoniste des récepteurs du PAF. Les PMN s'accrochent donc au PAF présent à la surface des HUVEC via leurs récepteurs du PAF, ce qui provoque l'activation des intégrines CD18/CD l lb exprimées par les PMN [19]. Celles-ci interagissent avec ICAM-1 (intercellular adhesion molecule-1) exprimée de manière constitutive par les HUVEC et stabili- 


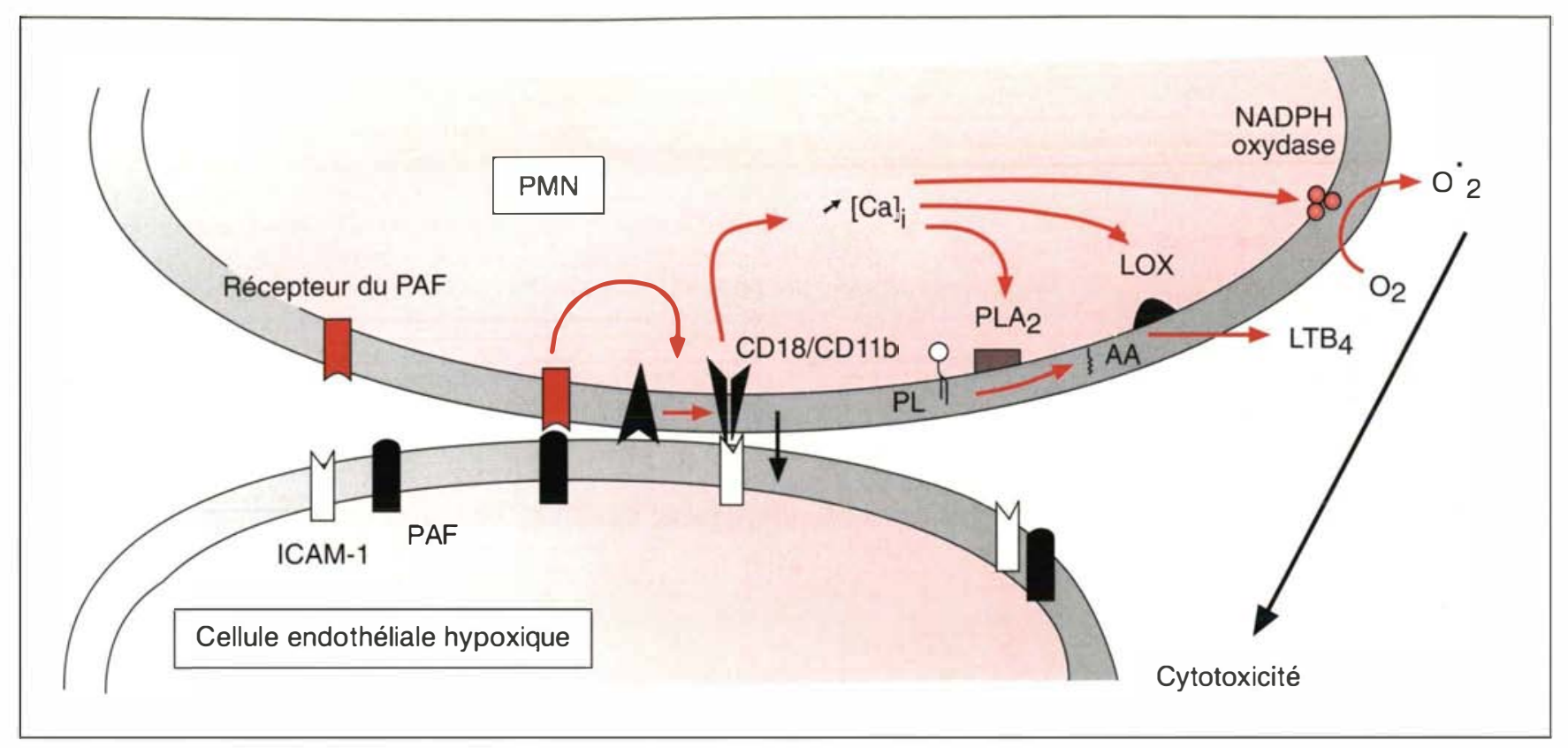

Figure 3. Interaction entre cellules endothéliales hypoxiques et polymorphonucléaires neutrophiles (PMN). Le PAF (platelet-activating factor), exprimé à la surface de la cellule endothéliale hypoxique, interagit avec son récepteur, exprimé à la surface des $P M N$, ce qui provoque l'activation des intégrines $C D 18 / C D 11 b$ qui se lient à la molécule d'adhérence endothéliale ICAM-1 (intercellular adhesion molecule-1). Les PMN s'activent en adhérant aux cellules endothéliales: I'élévation de [C $\left.\mathrm{Ca}^{2+}\right]$ i stimule l'activité de $P L A_{2}$ (phospholipase $\mathrm{A}_{2}$ ), des lipoxygénases (LOX) et de la NADPH oxydase, avec pour conséquence la production de molécules inflammatoires, leucotriènes et radicaux libres. $P L$ : phospholipide, $A A$ : acide arachidonique, $L T B_{4}$ : leucotriène $B_{4^{\prime}} O_{2} \bullet$ : radical superoxyde.

sent l'adhérence des PMN aux HUVEC. ICAM-1 est une molécule d'adhérence exprimée à la surface de nombreuses cellules. C'est une glycoprotéine appartenant à la famille des immunoglobulines et qui sert de ligand pour les intégrines exprimées par les leucocytes [11]. Cette interaction est nécessaire pour que l'adhérence soit mesurable puisqu'on peut l'inhiber par des anticorps dirigés contre l'une de ces différentes molécules d'adhésion et ce, même lorsque le PAF est présent à la surface des HUVEC [18] .

L'adhérence des PMN aux cellules endothéliales activées par l'hypoxie conduit aussi à une activation des PMN eux-mêmes, par suite de la liaison entre CD18/CD1 lb et ICAM-1. On peut ainsi observer une augmentation de la concentration calcique cytosolique dans ces PMN adhérents. Ils relarguent aussi une grande quantité d'anions superoxydes, ce $\mathrm{m} / \mathrm{s} n^{\circ} 8 / 9 \mathrm{vol}$. 10, août-septembre 94 qui implique l'activation de la NADPH oxydase et synthétisent du leucotriène $B_{4}$ dû à l'activation de la $\mathrm{PLA}_{2}$ et de la 5-lipoxygénase [20]. L'activation de ces différentes enzymes est probablement liée à l'augmentation de la concentration cytosolique en calcium. Une des conséquences importantes de cette activation des PMN est qu'ils induisent une cytotoxicité au niveau des HUVEC auxquelles ils adhèrent : cette cytotoxicité est relayée par les radicaux libres sécrétés par les PMN [20].

Ces différents résultats montrent que l'hypoxie perturbe fortement les interactions "pacifiques" normales entre cellules endothéliales et PMN et engendre ainsi une situation typiquement inflammatoire avec adhérence et activation des PMN. Ces différentes interactions entre ces deux types cellulaires sont résumées dans la figure 3.
Un troisième type cellulaire peut également être impliqué dans ce processus : il s'agit des cellules musculaires lisses (CML) qui, dans la paroi veineuse, sont sous-jacentes aux cellules endothéliales. Les interactions entre ces deux types cellulaires ont été étudiées de manière indirecte en examinant l'influence de milieux conditionnés par des HUVEC maintenues sous hypoxie sur la prolifération de CML in vitro. Le milieu conditionné est obtenu en incubant des HUVEC sous hypoxie pendant deux heures et en reprenant le milieu d'incubation. Ce milieu est alors ajouté aux CML et leur prolifération est mesurée au cours du temps. Une activité proproliférative pour les CML a pu être mise en évidence en présence du milieu conditionné par les HUVEC sous hypoxie par comparaison au milieu de cellules témoins [21]. Elle est le résultat de l'activation des 


\section{RÉFÉRENCES}

29. Galt SW, Zwolak RM, Wagner RJ, Gilbertson JJ. Differential response of arteries and vein grafts to blood flow reduction. J Vasc Surg $1993 ; 17$ : S63-70.

30. Kuchan MJ, Frangos JA. Shear stress regulates endothelin-P release via protein kinase $C$ and $c G M P$ in cultured endothelial cells. Am J Physiol 1993 ; 264 : H150-6.

31. Luscher TF, Tanner FC. Endothelial regulation of vascular tone and growth. $A m$ $J$ Aypertens 1993 ; 6 : 283S-93S.

32. Michiels C, Arnould T, Houbion A, Remacle J. Etude comparative de l'effet protecteur des différents phlébotoniques sur les cellules endothéliales en hypoxie. Phlébologie 1991 ; 44 : 779-86.

33. Arnould T, Michiels C, Remacle J. Effet du Ginkor Fort sur l'activation des cellules endothéliales induites par une hypoxie sévère. Act Vasc Intern 1992 ; 4 : 40-7.

34. Janssens D, Michiels C, Arnould T, Remacle J. Effets comparés du Ginkor Fort, de la troxérutine, de l'Esbériven sur l'activation des cellules endothéliales induites par une hypoxie sévère. Angiologie $1993 ; 11: 3-7$.

35. Michiels C, Arnould T, Alexandre I, Houbion A, Remacle J. Effect of naftidrofuryl on hypoxia-induced activation and mortality of human endothelial cells. $J$ Pharmacol Exp Ther 1993 ; 267 : 904-11.

36. Scott HJ, Cheatle TR, McMullin GM, Coleridge-Smith PD, Scurr JH. Reappraisal of the oxygenation of blood in varicose veins. $B r J$ Surg $1990 ; 77: 9346$.

37. Cornwall CV, Dore CJ, Lewis JD. Leg ulcer : epidemiology and aetiology. Br J Surg $1986 ; 73: 693-6$.

38. Lengyel I, Acsady G. Histomorphological and pathobiochemical changes in varicose veins. A possible explanation of the development of varicosis. Acta Morphol Hung $1990 ; 38: 259-67$.

39. Crotty TP. The origin and the progression of varicose veins. Med Hypotheses $1993 ; 37: 198-204$

40. Second European Consensus Document of Chronic Critical Leg Ischemia. Circulation $1991 ; 84$ : IV1-IV26.

41. Thomas PRS, Nash GB, Dormandy JA. White cell accumulation in dependent legs of patients with hypertension : a possible mechanism for trophic changes in the skin. Br Med J 1988 ; 296 : 1963-5.

42. Coleridge-Smith PD, Thomas $P$, Scurr JH, Dormandy JA. Causes of venous ulceration : a new hypothesis. $B r M e d J$

HUVEC par l'hypoxie car, lorsque l'activation de ces cellules est bloquée par le B-hydroxybutyrate, on n'observe plus de prolifération des CML. Diverses expériences réalisées en présence de différents inhibiteurs ont permis de démontrer que les prostaglandines synthétisées en grande quantité par les HUVEC incubées sous hypoxie étaient en partie responsables de cette activité mitotique. La PGF $_{2 \alpha}$ a l'effet le plus important. Elle agit en synergie avec un autre facteur, qui n'est ni une protéine synthétisée de novo, ni le PAF, mais probablement le bFGF (basic fibroblast growth factor) [21].

\section{Relation entre les caractéristiques des veines variqueuses et le mécanisme d'activation des cellules endothéliales par I'hypoxie}

Ces différents résultats montrent clairement que les cellules endothéliales peuvent être stimulées in vitro par l'hypoxie, une situation qui mime l'ischémie qui se produit in vivo dans les vaisseaux sanguins durant la stase veineuse : l'ischémie, occasionnée par un arrêt de la circulation sanguine, entraîne une diminution de l'apport en oxygène et en nutriments au tissu normalement irrigué et prévient l'élimination des déchets métaboliques et du $\mathrm{CO}_{2}$. Par ailleurs, l'hypoxie que nous réalisons in vitro n'entraîne qu'une diminution de la pression partielle en oxygène. Le modèle expérimental est donc réductionniste, mais il a l'avantage de pouvoir disséquer l'influence de chaque paramètre, ici le rôle de l'oxygène, sur une population pure de cellules. Il reste cependant tout à fait pertinent car de nombreuses études sur des organes isolés ou perfusés in situ ont montré que la diminution de la $\mathrm{PO}_{2}$ est l'acteur principal des dommages occasionnés par une ischémie. L'activation des cellules endothéliales par l'hypoxie initie une cascade d'événements impliquant neutrophiles et cellules musculaires lisses qui peut, lorsqu'elle est transposée in vivo, expliquer le développement de veines variqueuses. L'adhérence des PMN aux cellules endothéliales n'est que la première étape du processus de diapédèse qui conduit à l'infiltration des PMN dans l'intima et dans la média des veines [22]. Ces PMN sont aussi stimulés et peuvent relarguer diverses molécules actives telles que des radicaux libres et des icosanoïdes. Ces médiateurs, en conjonction avec ceux libérés par les cellules endothéliales elles-mêmes, se retrouvent dans la paroi veineuse où ils peuvent induire différentes réactions similaires aux diverses altérations tissulaires observées dans les veines variqueuses.

$\mathrm{Au}$ moins cinq caractéristiques principales des parois des veines variqueuses peuvent être analysées à la lumière de cette hypothèse [23].

(1) Dans la paroi des veines variqueuses, la barrière endothéliale est toujours présente alors que d'autres cellules comme les CML subissent de profonds changements. Nous avons expérimentalement montré qu'une hypoxie sévère de deux heures in vitro induit de profonds changements métaboliques au sein des cellules endothéliales : il se produit une activation avec synthèse accrue de médiateurs de l'inflammation et de facteurs de croissance. Cependant, la viabilité de ces cellules est préservée et les cellules endothéliales retrouvent leur homéostasie dès qu'elles sont remises dans des conditions normoxiques [15]. Cet effet de l'hypoxie permet d'expliquer le recrutement des neutrophiles et la prolifération des cellules musculaires lisses alors que la barrière endothéliale reste intacte. Ce tableau est opposé à celui qu'on peut observer dans l'athérosclérose où l'endothélium est abîmé et où les altérations de la paroi artérielle sont liées à l'intervention des plaquettes et des monocytes.

(2) Une grande quantité d'enzymes lysosomiales est retrouvée au sein des couches cellulaires sous-jacentes aux cellules endothéliales dans les veines variqueuses par rapport aux veines normales [23]. Ces enzymes sont relarguées par les PMN activés. Leur présence dans le tissu veineux variqueux peut donc être facilement expliquée puisque nous avons montré expérimentalement que les PMN adhèrent aux cellules endothéliales activées par l'hypoxie, ce qui entraî- 
ne leur propre activation. On peut également penser que leur infiltration dans le tissu sous-jacent est aussi une conséquence de cette adhérence, ce qui a d'ailleurs été montré dans d'autres modèles expérimentaux. Une fois dans la média et par suite de leur activation, ils y relargueraient leurs protéases par dégranulation. Des expériences réalisées sur une veine ombilicale perfusée en hypoxie montrent effectivement l'infiltration de neutrophiles sous l'endothélium et leur activation, lorqu'ils adhèrent à l'endothélium, est attestée par la présence de nombreux replis membraneux à la surface du globule blanc (figure 4; Arnould, résultats non publiés). Par ailleurs, on n'observe que très peu de PMN adhérant à l'endothélium d'une veine maintenue en normoxie et ces neutrophiles ne sont pas activés

(3) Le collagène et les protéoglycanes sont altérés dans la paroi des veines variqueuses : on y retrouve, par exemple, une proportion augmentée de collagène soluble. Les radicaux libres sont responsables de la fragmentation de ces deux types de

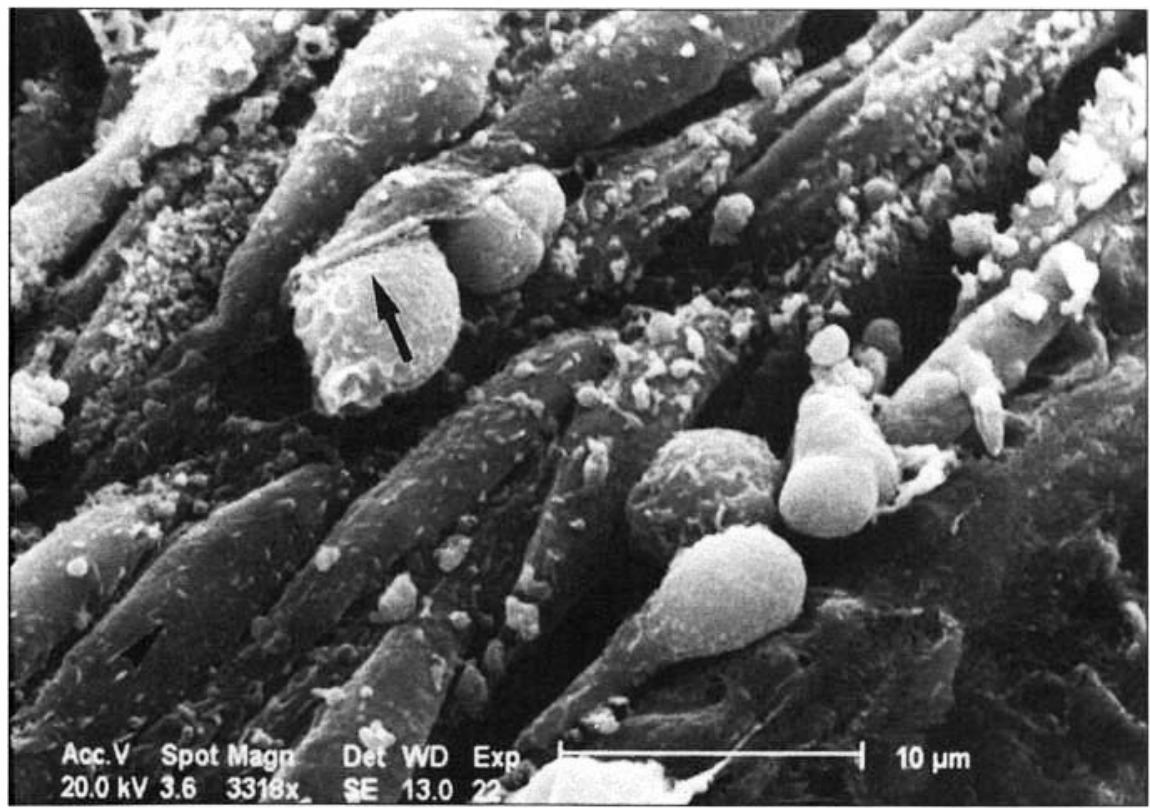

Figure 4. Micrographie en microscopie électronique à balayage de la surface luminale de l'endothélium d'une veine ombilicale humaine maintenue sous hypoxie pendant 2 heures et perfusée ensuite avec des neutrophiles humains non activés pendant 5 minutes. La tête de flèche indique une cellule endothéliale tandis que la flèche montre un PMN s'infiltrant par diapédèse sous l'endothélium (grossissement $=3318 X$ ). de matrice extracellulaire et notamment de collagène, sont prolifératives et perdent l'expression de la myosine et de l' $\alpha$-actine [26, 27]. Cette modulation phénotypique pourrait également être le résultat de l'action de médiateurs relargués par les cellules endothéliales activées par l'hypoxie. La prolifération des CML [20], ainsi que leur capacité accrue de synthèse des composés de la matrice extracellulaire [28], peuvent donc facilement rendre compte de l'épaississement de la paroi veineuse pathologique.

(5) Finalement, la prolifération des CML, les changements qualitatifs et quantitatifs dans la synthèse des protéines de la matrice extracellulaire ainsi que l'hydrolyse partielle de ces protéines par les molécules relarguées par les PMN infiltrés expliquent la désorganisation totale de la paroi vasculaire observée dans les veines variqueuses. La figure 5 présente un schéma de ces différentes altérations. Cette désorganisation, en plus de la perte de la contractilité des CML, conduit à des altérations du tonus vasculaire.

Nous n'avons pas tenu compte dans ce processus de l'effet direct de l'hypoxie sur les PMN eux-mêmes. Certains résultats préliminaires suggèrent que l'hypoxie pourrait activer directement les PMN. En revanche, les CML semblent très résistantes aux effets de l'hypoxie (De Leener, communication personnelle). De plus, d'autres cellules sanguines, telles que les plaquettes, les monocytes ou les lymphocytes, connues pour leur réaction en présence de PAF et d'autres médiateurs relargués par les cellules endothéliales activées par l'hypoxie, peuvent aussi être impliquées dans ce processus et amplifier les altérations tissulaires.

Un autre paramètre important dans la stase veineuse est la diminution du flux sanguin et donc des forces de cisaillement au niveau de l'endothélium [29]. Une diminution de ces forces affecte la production de nombreux métabolites par les cellules endothéliales, comme par exemple celle de l'endothéline [30], de la prostacycline, de l'EDRF, du PDGF... Il a été montré in vivo qu'une diminution locale du flux sanguin entraînait un remodelage des artères mais 


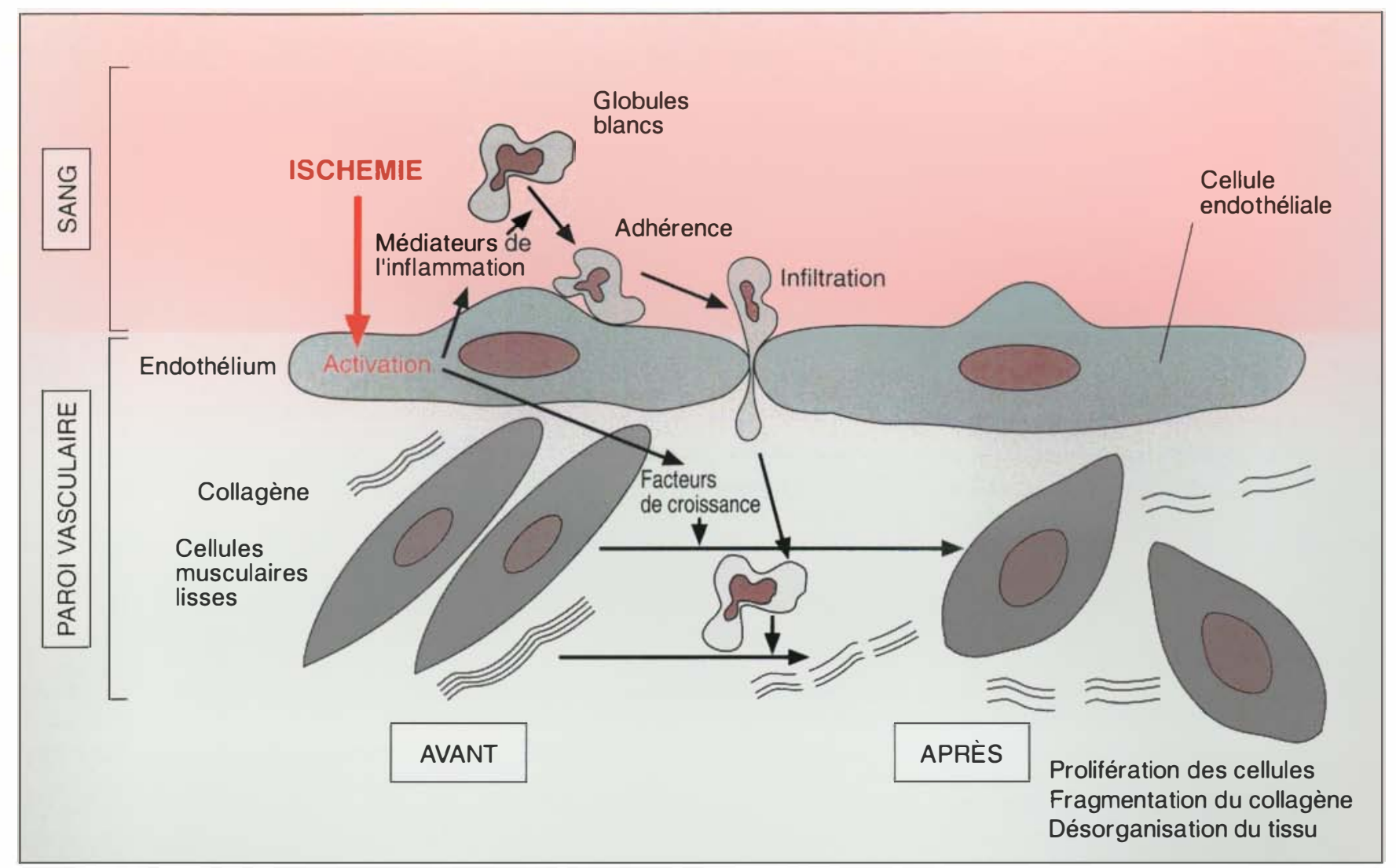

Figure 5. Désorganisation de la paroi d'une veine soumise à l'ischémie. L'activation des cellules endothéliales et leurs interactions avec les polymorphonucléaires neutrophiles entraînent une désorganisation et une altération de la paroi vasculaire caractérisées par une prolifération des cellules musculaires lisses qui perdent leurs propriétés contractiles, des altérations de la matrice extracellulaire (collagène et protéoglycanes) sous l'effet des anions superoxydes et des protéases.

aussi des veines [31] avec épaississement de la paroi vasculaire. Des changements de production de différents métabolites au niveau de l'endothélium, induits par l'effondrement des forces de cisaillement lors de la stase veineuse, pourraient aussi participer, avec l'hypoxie, à la pathogénie de la maladie variqueuse.

Cette cascade d'interactions peut, bien sûr, être arrêtée et les altérations réparées, mais, si elle se répète, elle pourrait conduire à des changements irréversibles dans la paroi vasculaire.

\section{Effet de médicaments sur l'activation des cellules endothéliales par I'hypoxie}

Même si l'intérêt clinique des médi- caments phlébotoniques sur la survenue et l'évolution de l'insuffisance veineuse n'est pas établi, il nous semblait intéressant d'en étudier les potentialités. Nous avons étudié l'effet de deux médicaments sur la cascade provoquée in vitro par l'hypoxie sur la cellule endothéliale et son interaction avec les neutrophiles. Deux médicaments furent particulièrement étudiés et se sont avérés très efficaces : l'extrait de Ginkgo biloba et le naftidrofuryl, qui ont des effets inhibiteurs in vitro aux concentrations mesurées dans le sang lors de leur usage thérapeutique. Ces deux préparations peuvent inhiber fortement, et avec un effet dépendant de la dose, les différents événements de la cascade d'activation des cellules endothéliales présentés sur la figure 2, c'est-àdire la chute de la concentration en
ATP, l'élévation de la concentration calcique, l'activation de la phospholipase $A_{*}$, la synthèse de prostaglandines et de PAF [31-35]. Une inhibition de l'adhérence des PMN a pu également être mise en évidence. C'est en prévenant la chute de la concentration en ATP, probablement en préservant la fonction respiratoire des mitochondries au début de l'hypoxie, que ces substances préviennent les événements subséquents de la cascade d'activation des cellules endothéliales sous hypoxie et donc aussi l'augmentation de leur pouvoir adhésif vis-à-vis des PMN. Ces résultats indiquent clairement une action protectrice importante de ces médicaments sur l'endothélium soumis à une hypoxie, ce qui pourrait apporter une explication rationnelle d'un effet thérapeutique potentiel. 


\section{Conclusion}

$\mathrm{Si}$ les mesures comparatives de $\mathrm{TcPO}_{2}\left(\mathrm{PO}_{2}\right.$ transcutanée), chez des patients atteints d'insuffisance veineuse et des personnes saines, dans différents conditions, sont nombreuses, elles ne donnent cependant pas d'indication sur l'état d'oxygénation de la paroi vasculaire des veines des membres inférieurs. Très peu de données sont disponibles sur la $\mathrm{PO}_{2}$ du sang dans les veines variqueuses. Même si la stase veineuse, entraînant une diminution de l'apport en oxygène, a été à l'origine des altérations de la veine présentées dans cet article, cela ne signifie pas pour autant que les tissus de la veine variqueuse présentent de faibles $\mathrm{PO}_{2}$ en dehors de cette stase. Au contraire, Scott et al. [36] ont montré qu'en position couchée, la $\mathrm{PO}_{2}$ du sang de veines variqueuses était plus élevée que celle de veines normales. Cette différence pourrait être due à la présence d'anastomoses artérioveineuses, peut-être issues d'un mécanisme de compensation chez les patients atteints d'insuffisance veineuse. Cependant, après 30 minutes en position debout, la $\mathrm{PO}_{2}$ chute fortement dans les deux cas et on n'observe plus de différence entre veine malade et veine saine : la diminution moyenne de la $\mathrm{PO}_{2}$ est de $18 \%$ pour les veines saines et de $35 \%$ pour les veines malades. Cette diminution de $\mathrm{PO}_{2}$ pendant la stase veineuse, associée au reflux veineux, et à un terrain génétique prédisposé, pourrait être suffisante pour déclencher le processus d'activation des cellules endothéliales et la cascade d'événements qu'elle entraîne.

On sait par ailleurs que l'ischémie est une situation associée au développement des veines variqueuses [37-39] ; le "Deuxième document consensus européen sur l'ischémie critique des jambes "[40] stipule que les perturbations métaboliques au niveau de l'endothélium entraînées par l'ischémie activent la microcirculation, sans que le mécanisme biochimique soit connu. De plus, Thomas et al. [41] ont mis en évidence une accumulation de leucocytes dans les jambes de patients souffrant d'insuffisance veineuse chronique. Coleridge-Smith et al. [42] proposèrent que l'obstruction $\mathrm{m} / \mathrm{s} n^{\circ} 8 / 9$ vol. 10, août-septembre 94 des capillaires par des leucocytes pourrait contribuer à engendrer une ischémie locale et, par la suite, une ulcération induite par l'activation des leucocytes et les dommages des cellules endothéliales résultant de l'ischémie qui est effectivement observée chez les malades. Dans l'hypothèse développée ici, nous proposons que l'ischémie résultant de la stase veineuse est un facteur initiateur de l'activation des cellules endothéliales, responsable de l'activation des PMN. Les PMN ainsi activés peuvent alors être trappés dans les capillaires et causer une obstruction.

L'extrapolation du comportement des cellules endothéliales en culture soumises à une hypoxie sévère à celui de cellules in vivo est justifiée par le fait qu'on obtient les mêmes effets, c'est-à-dire l'activation des cellules endothéliales, avec synthèse de prostaglandines et recrutement et stimulation des PMN conduisant à leur adhérence et à leur infiltration, lorsqu'on soumet une veine ombilicale intacte à une ischémie (figure 3). Ces résultats montrent qu'on peut reproduire, sur une veine entière, ce qui a été observé sur les cellules endothéliales en culture ; on peut donc penser que les mêmes phénomènes peuvent se produire in vivo pendant une ischémie.

Cette hypothèse fournit pour la première fois un lien biochimique entre l'observation que la station debout prolongée est un facteur étiologique important dans l'apparition des varices et le développement de la maladie Cette cascade d'événements a lieu dans un environnement global où les prédispositions génétiques, aussi bien que des facteurs externes tels que mécaniques ou hormonaux, jouent aussi un rôle important. Par exemple, des perturbations mécaniques peuvent rendre la paroi veineuse plus fragile et pourraient également conduire à la prolifération des CML. Cette hypothèse est cependant intéressante car elle aborde le problème de la pathogénie des troubles veineux d'une façon nouvelle ; d'autres approches expérimentales pourront être développées pour la tester.

Notre modèle expérimental simple, qui découle de cette hypothèse, permet de tester et de développer de nouveaux médicaments basés sur une nouvelle stratégie thérapeutique. Nous avons déjà montré que certains médicaments phlébotoniques ou utilisés dans les processus ischémiques pouvaient effectivement inhiber la cascade déclenchée par l'activation hypoxique des cellules endothéliales. Cet effet ne signifie pas que la veine variqueuse va redevenir normale à la suite de la prise de tels médicaments, mais qu'ils pourraient être utiles lors de la stase proprement dite pour prévenir l'initiation de cette cascade. L'utilisation du modèle d'hypoxie cellulaire pour un premier criblage de nouvelles molécules pharmacologiques pourrait conduire à la mise au point de nouveaux médicaments dans la décennie à venir

\section{Remerciements}

Nous voudrions reconnaître le support du FNRS, du FRRC et du SSTC et remercier la clinique Saint-Elisabeth de Namur et l'Hôpital Notre Dame de Charleroi pour l'approvisionnement en cordons ombilicaux. Nous remercions aussi Lipha et les Laboratoires Beaufour pour leur soutien lors des études sur les médicaments.

\section{Summary}

Key role of hypoxia and endothelial cells in the pathogenesis of varicose veins

Varicose veins are a very common pathology but its etiology still remains not well understood. Epidemiological studies have shown that, in addition to genetic and hormonal factors, prolonged standing position may contribute to the development of this pathology. However, the relation between the standing position and the physiopathological modifications typical of the varicose veins is still unclear. In this article, we sum up experimental data obtained from the study of the effects of hypoxia on endothelial cells et we examine how these results can explain the alterations observed in pathological vein and the action of phlebotonic drugs. All these data led to a coherent hypothesis for one possible cause of the chronic venous insufficiency coming from a local ischemia. 